\title{
Recent results on forward physics and jets at LHC
}

\author{
Grzegorz Brona ${ }^{1, a}$ for the ATLAS and CMS Collaborations \\ ${ }^{1}$ University of Warsaw, Faculty of Physics, Warsaw, Poland
}

\begin{abstract}
An overview of the recent results on forward physics from the LHC Run2 is presented. Both CMS and ATLAS results obtained in proton-proton collisions at $\sqrt{s}=13 \mathrm{TeV}$ are shown. Forward energy flow measured at high pseudorapidity, inclusive jets, and jets reconstructed in CMS-CASTOR detector are discussed. In addition, results on the pseudorapidity spectra of charged particles are presented.
\end{abstract}

\section{Forward energy flow}

Forward energy flow is an observable that provides an independent measure of the underlying event activity (UE). It can be used to tune the models that simulate this component of an interaction. A good description of the UE is a prerequisity to study hard processes, which lay on top of the UE activity, and to look for new physics phenomena. Moreover it is an important background for precise measurements in QCD and EW domains. The measurement of the UE in new kinematical domains - at high energies and high pseudorapidities, gives also an insight into QCD dynamics at low scales. Finally, results on the forward energy flow can be used as input to the cosmic ray physics modelling.

At CMS [1] two measurements of the forward energy flow at high rapidities at $\sqrt{s}=13$ $\mathrm{TeV}$ are released. The first is performed with the CMS Hadronic Forward (HF) calorimeter within $3.15<|\eta|<5.20$, and the CASTOR calorimeter covering $-6.6<|\eta|-5.2$ [2], while the second only with CASTOR [3]. Both are done with low luminosity runs.

The analysis with HF and CASTOR is focused on two samples of events: an HF-OR sample defined with a requirement of at least one $\mathrm{HF}$ calorimeter tower above $5 \mathrm{GeV}$ at at least one side of CMS, and an HF-AND sample with a requirement of at least one HF tower above $4 \mathrm{GeV}$ at both sides of the CMS detector. The former is an inclusive sample while the latter is a non-single diffractive enhanced sample. The observable in the analysis is a sum over calorimeter towers in a given $|\Delta \eta|$ bin. The results are corrected for pile-up and detector noise. Finally, the energy distributions are corrected back to the particle level with corrections derived from Monte Carlo. The largest uncertainty arrives from the calorimeter global energy scale uncertainty, which in the studied region is in the range 10-17\%. In Fig. 1 and 2 energy flow distributions in HF-OR and HF-AND samples are presented, respectively. For the comparison PYTHIA8 with different tunes, and different diffractive component modeling is used. Also predictions of EPOS LHC and QGSJET-II, models for cosmic rays physics,

a e-mail: grzegorz.brona@fuw.edu.pl 

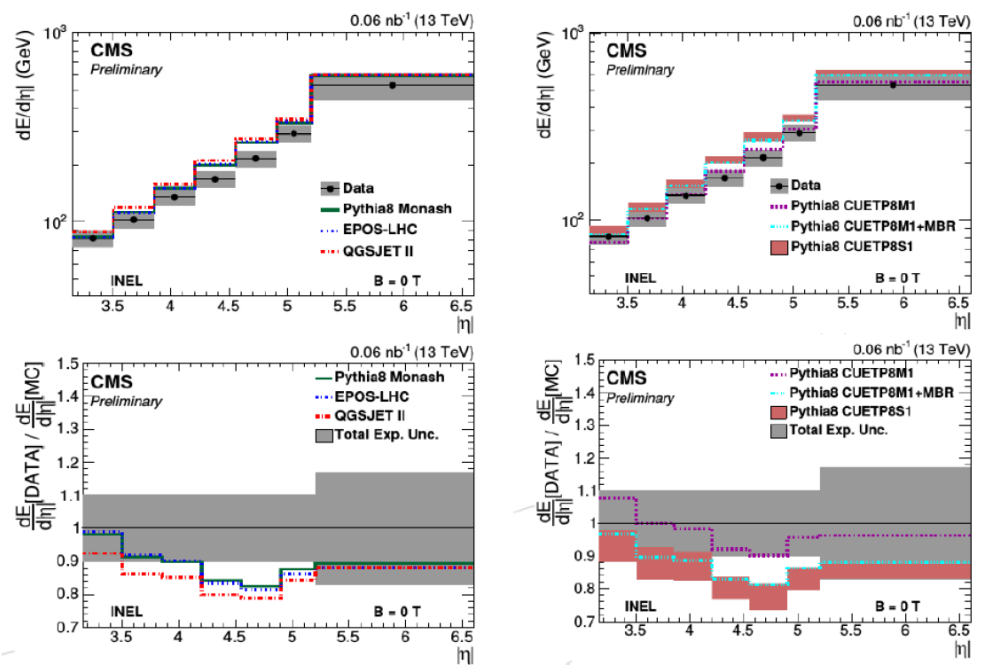

Figure 1. The inelastic forward energy flow distributions. On the left and right the same data points are presented while models differ. On the bottom plots ratios of the results to the predictions are presented [2].

are presented. The best agreement for the HF-OR sample for all the models is obtained in the lowest $|\eta|$ bins. Then for higher HF rapidities models overestimate the data. In the CASTOR bin the agreement is again better. Overall PYTHIA8 CUETP8M1 describes the HF-OR sample best. Predictions with different diffraction models (PYTHIA8 CUETP8M1 with Schuler-Sjöstrand, and with Minimum Bias Rockefeller models) exhibit large variations. For the HF-AND sample the spread between models tends to be smaller. Cosmic ray models are inside the experimental uncertainties, and provide a qualitatively good description. The best agreement among the PYTHIA tunes, is obtained with PYTHIA8 CUETP8M1. The HF-AND and HF-OR distributions do not exhibit significant differences in the spectrum shape.

The second analysis focuses on CASTOR, however the selection of events requires an activity exceeding $5 \mathrm{GeV}$ in at least one HF tower. The CASTOR calorimeter does not have a segmentation in $\eta$ but it is divided in 14 modules along the $z$ direction and 16 sectors in $\phi$. The first two modules are electromagnetic modules, and the rest hadronic. Three observables are defined: total energy recorded in CASTOR per event, total energy deposited in the electromagnetic modules per event, and total energy in the hadronic modules. The dominant uncertainty is again the energy scale uncertainty, $17 \%$. Distributions are corrected to the stable particle level with Monte Carlo models. In Fig. 3 the distributions are plotted. In the first bins diffractive events form a distinctive peak. In the soft part of the spectrum (up to energy deposits of a few hundred GeV) PYTHIA8 and HERWIG tend to overestimate the data. The observables are sensitive to the PYTHIA8 tuning, none of the models and tunes is able to describe all three observables in the whole spectrum of the energy. 

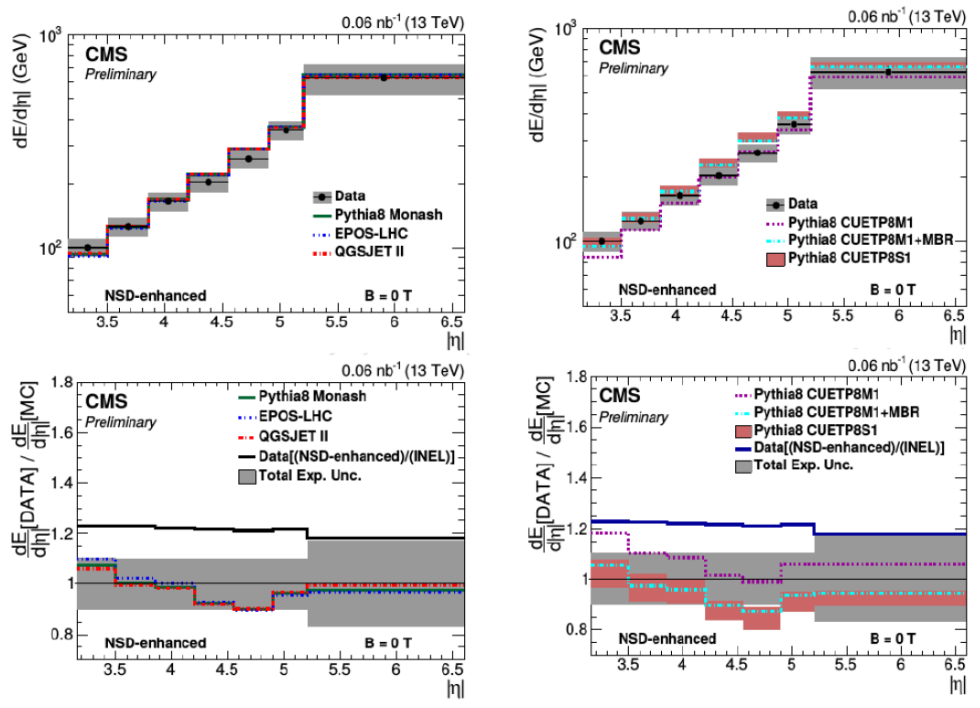

Figure 2. The NSD-enhanced forward energy flow distributions. On the left and right the same data points are presented while models differ. On the bottom plots ratios of the results to the predictions are presented [2].

\section{Jets}

Both experiments, CMS and ATLAS [4], performed measurements of the inclusive jets cross sections at $13 \mathrm{TeV}[5,6]$. The cross sections are obtained in bins of rapidity. In case of CMS seven bins are defined covering a range up to $|y|=4.7$, and $p_{T}=2000 \mathrm{GeV}$ for most central jets. In ATLAS the range is smaller, up to $y=3.0$ (six bins) but the coverage in $p_{T}$ is wider, up to $3200 \mathrm{GeV}$. The analysis in CMS makes use of the anti- $k_{T}$ algorithm with two different jet radii $R=0.4$ and 0.7 . The better agreement with the NLO QCD predictions (obtained with NLOJet ++ ) are obtained for a smaller $R$. In case of ATLAS only $R=0.4$ is used. The data are compared with NLOJet ++ with different PDF sets - CT14, MMHT2014, NNPDF3.0, CT10, HERAPDF2.0 and ABM12. A ratio of theory predictions to the data is presented in Fig. 4. All predictions describe the data overall well. In the forward region at large $p_{T}$ there is a tendency in the theoretical approach to overestimate the data. Predictions with the ABM12 PDF set have a tendency to underestimate the data in medium and low rapidity bins.

The analysis of jets reconstructed within the CASTOR acceptance is based on $0.212 \mathrm{nb}^{-1}$ of integrated luminosity recorded in a condition of very low pile-up (6\% of probability of an interaction per bunch crossing) [7]. Jets are reconstructed with the anti- $k_{T}$ algorithm with $R=0.5$ in a range of $p_{T} 3-13 \mathrm{GeV}$. Very forward jets are sensitive to the low- $x$ gluon PDF, and to non-linear effects. In Fig. 5 the inclusive cross section of jets in CASTOR is plotted versus $p_{T}$. Four different sources of systematics are shown. These are: CASTOR energy scale uncertainty (CES), luminosity uncertainty, CASTOR true acceptance (the position of the detector varies during data taking and is known with a precision of $2 \mathrm{~mm}$ ), and modeling of the unfolding corrections. All tested models are in agreement with the data within the systematic uncertainty band. The spectrum has only a moderate sensitivity to the underlying 

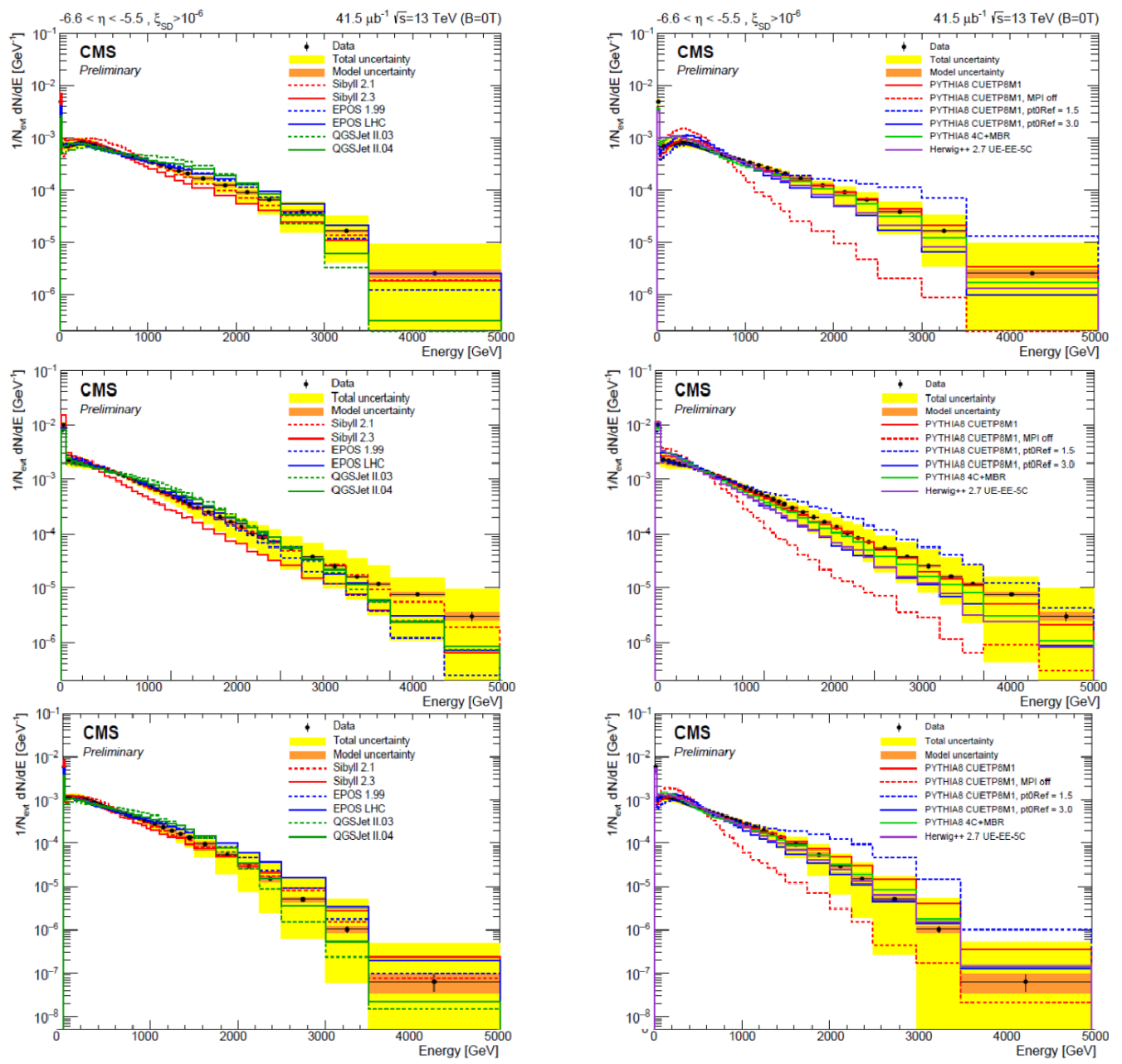

Figure 3. From top to bottom: total energy recorded in CASTOR per event, total energy deposited in the electromagnetic modules per event, and total energy deposited in the hadronic modules. On the left and right plots the same data points are presented while models differ [3].

PDF set of the model (Fig. 5 right). However, switching off the multiple parton interaction mechanism shifts the predicted spectrum significantly, and causes deviation from the data.

\section{Charged particles spectra and UE with leading track/jet}

Charged particles spectra are measured both by CMS and ATLAS [8-10]. At ATLAS two samples of events with a single primary vertex are selected: with at least 1 (2) tracks within $|\eta|<2.5$ and with $p_{T}>500 \mathrm{MeV}(100 \mathrm{MeV})$. Then primary charged-particle multiplicities as a function of pseudorapidity are calculated. The distributions are compared to model predictions: the EPOS LHC Monte Carlo fits data best in both samples. To compare with 

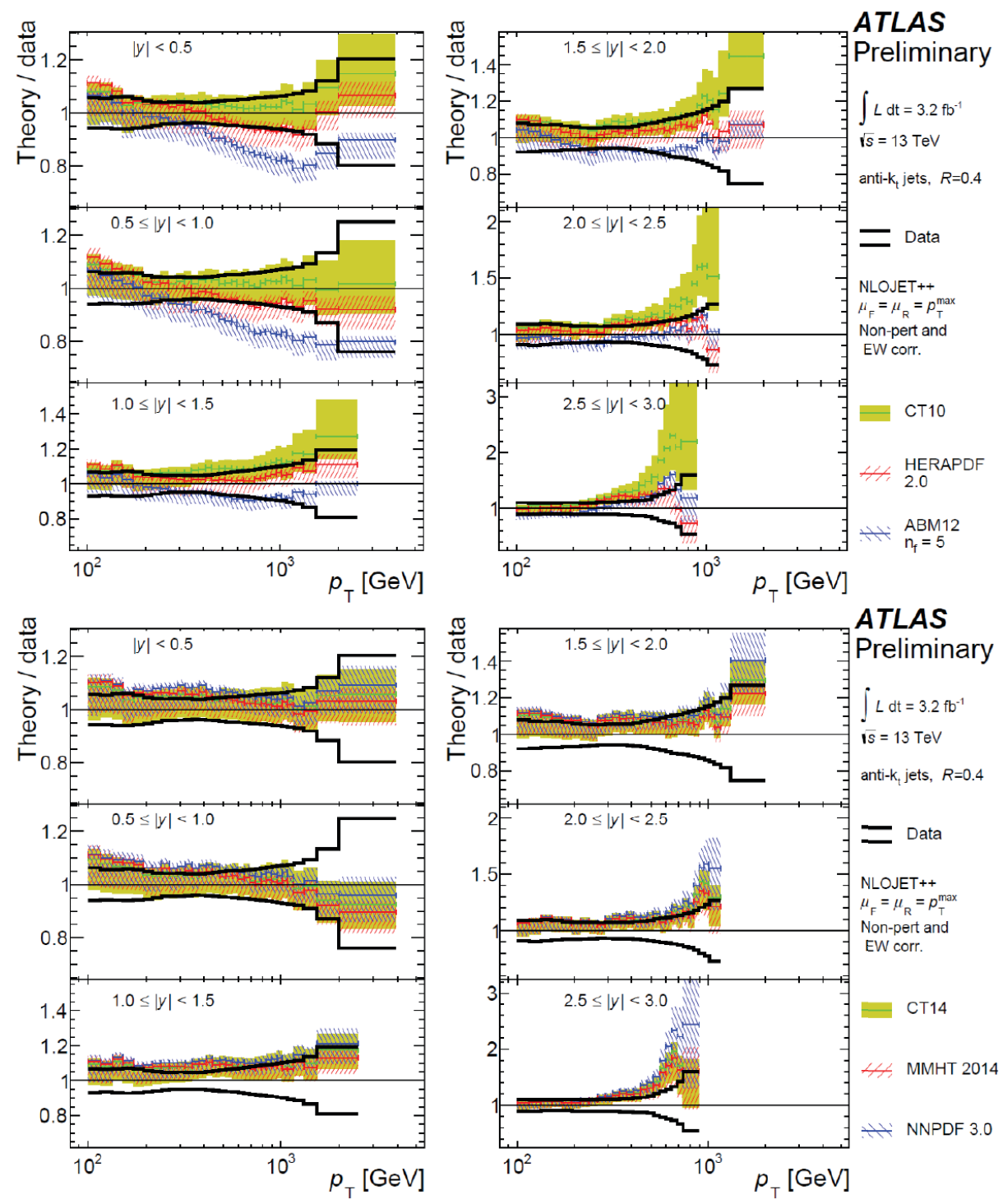

Figure 4. Ratio of the theoretical predictions for the inclusive jets cross section to the data. The plots are obtained for different bins in rapidity. As a model NLOJet ++ is used with various PDF sets [6].

previous results the range under study is restricted to $|\eta|<0.2$ and presented in Fig. 6. A $20 \%$ increase of the multiplicity is observed when moving from 7 to $13 \mathrm{TeV}$ center-of-mass energy. All models follow the data while the best description is obtained with EPOS LHC Monte Carlo. 

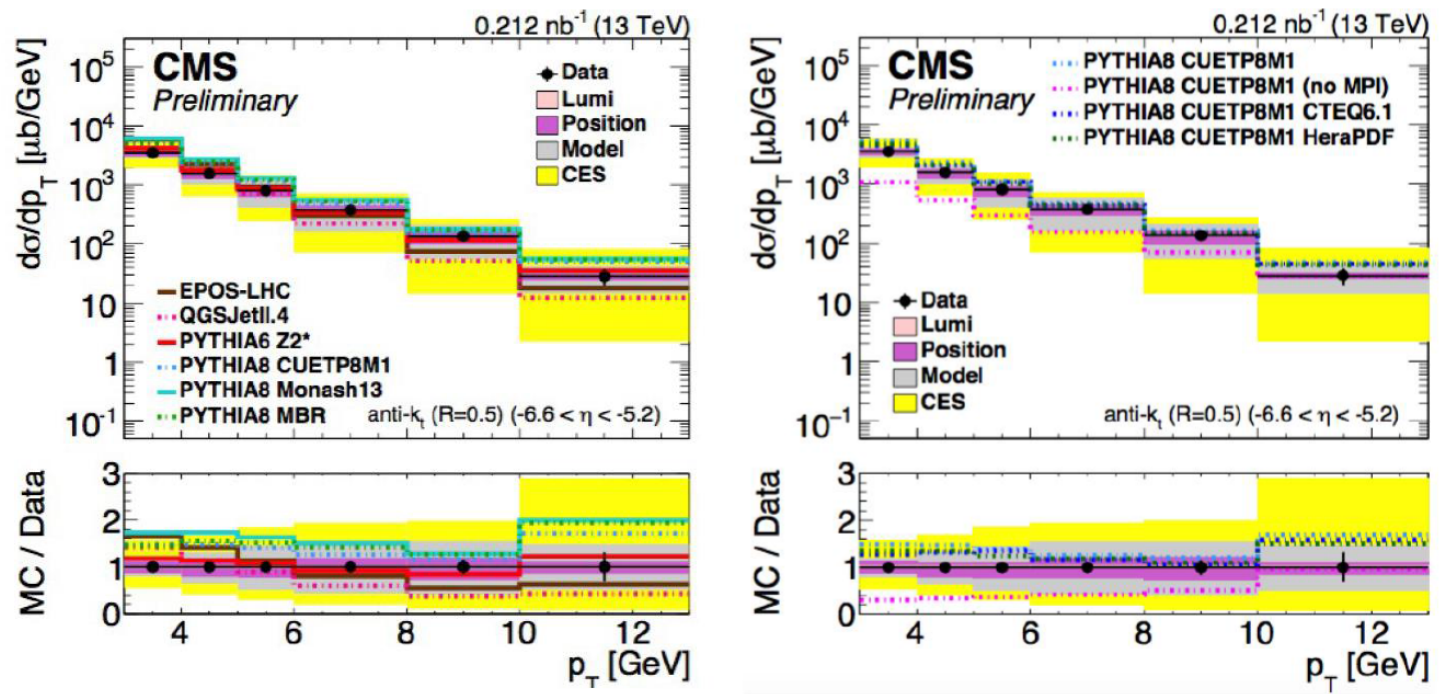

Figure 5. Cross section for the production of jets within $-6.6<|\eta|<-5.2$. Left and right plots present the same data, while models are different [7].

In case of the CMS analysis, basic selection of events is similar to ATLAS. An event enters the study if at least one charged particle with $p_{T}>500 \mathrm{MeV}$ is registered within $|\eta|<2.4$ limit. Then three subsamples are defined: 1) inelastic event sample with at least one particle with $E>5 \mathrm{GeV}$ in at least one $\mathrm{HF}$ detector, 2) non-single-diffractive (NSD) enhanced sample with at least one particle with $E>5 \mathrm{GeV}$ in both HF detectors, 3) single diffractive (SD) sample defined in the same way as the inelastic sample but with an additional veto on activity in the second HF. Distributions of the multiplicities are presented in Fig. 7 . In the inelastic sample the best agreement with data is obtained with EPOS LHC, HERWIG++ and PYTHIA8 CUETP8M1 models. The last from these models describes also the NSD sample well. The SD distribution is not described by any of the presented models.

\section{Acknowledgements}

This work was supported by Polish National Science Center, under contract DEC2012/07/E/ST2/01406.

\section{References}

[1] CMS Collaboration, JINST 3 S08004 (2008)

[2] CMS Collaboration, CMS-FSQ-15-006

[3] CMS Collaboration, CMS-FSQ-16-002

[4] ATLAS Collaboration, JINST 3435 (2008) S08003

[5] CMS Collaboration, Eur. Phys. J. C 76, 451 (2016)

[6] ATLAS Collaboration, ATLAS-CONF-2016-092 


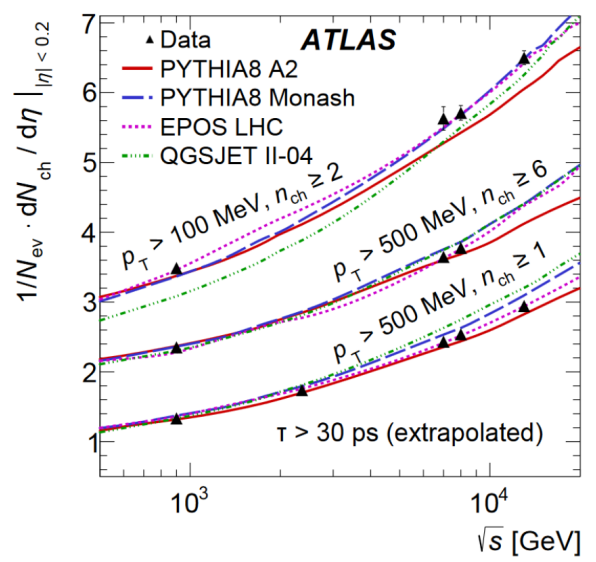

Figure 6. Charged particles multiplicity within $|\eta|<0.2$ for various energies [10]. The results were extrapolated to include particles with mean lifetime $\tau>30$ ps.
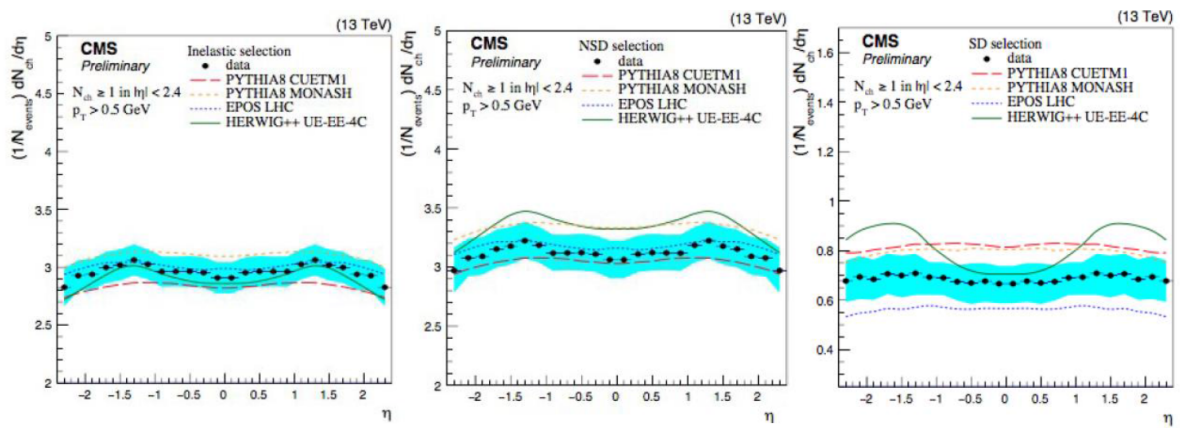

Figure 7. Multiplicity distribution in CMS at $\sqrt{s}=13 \mathrm{TeV}$ calculated in three samples: inclusive sample (left), non-single-diffractive enhanced sample (middle), single diffractive sample (right) [8].

[7] CMS Collaboration, CMS-FSQ-16-003

[8] CMS Collaboration, CMS-FSQ-15-008

[9] CMS Collaboration, Phys. Lett. B 758, 67 (2016)

[10] ATLAS Collaboration, Eur. Phys. J. C 76, 502 (2016) 